\title{
Influences of tactile-dot height and tip radius of curvature on the operational performance of cellular phones ${ }^{1}$
}

\author{
Wataru Toyoda ${ }^{\mathrm{a},{ }^{*},}$, Kouki Doi ${ }^{\mathrm{b}}$ and Hiroshi Fujimoto ${ }^{\mathrm{c}}$ \\ ${ }^{a}$ Graduate of Human Sciences, Waseda University, 2-579-15 Mikajima, Tokorozawa City, Saitama Prefecture, \\ 359-1192, Japan, tel.+81-4-2949-8113 ext.3625,E-mail: wataru_toyoda@toki.waseda.jp \\ ${ }^{\mathrm{b}}$ Department of Teacher Training and Information, National Institute of Special Needs Education, 5-1-1 Nobi, \\ Yokosuka City, Kanagawa Prefecture 239-8585, Japan, tel.+81-46-839-6849,E-mail: doi@nise.go.jp \\ ${ }^{\mathrm{c}}$ Faculty of Human Sciences, Waseda University, 2-579-15 Mikajima, Tokorozawa City, Saitama Prefecture, 359- \\ 1192, Japan, tel.+81-4-2949-8113 ext.3617,E-mail: fujimoto@waseda.jp
}

\begin{abstract}
Tactile dots located on operation keys of consumer products such as cellular phones contribute to improving accessibility for older people and people with visual impairment. The Japanese Standards Association (2000) and the International Organization for Standardization (2011) standardized tactile dots. However, reliable data on the appropriate sizes and the shapes was not necessarily available. The purpose of this study is to evaluate influences of the height $(0.1,0.3,0.55$, and 0.75 $\mathrm{mm})$ and the tip radius of curvature $(0.1,0.3,0.5,0.7$, and $0.9 \mathrm{~mm})$ of tactile dots on the operational performance of cellular phones in younger and older people. Sighted younger and older participants, whose hand was covered by a curtain, operated cellular phones with a tactile dot on its key 5 and without a tactile dot. As the result, both participants performed better at a particular height with larger tip radius of curvature. Furthermore, older participants operated better at high dots like 0.55-0.75 $\mathrm{mm}$. In contrast, younger participants performed better at $0.3 \mathrm{~mm}$ and relatively poorly at $0.1 \mathrm{~mm}$ and $0.75 \mathrm{~mm}$. Thus, comparatively high tactile dots are useful for improving the accessibility of products for the older and there is an appropriate height range for the younger.
\end{abstract}

Keywords: Accessibility, ISO 24503, JIS S 0011, Consumer products, Operational performance

\section{Introduction}

Tactile dots (dot-shaped tactile symbols) and tactile bars (bar-shaped tactile symbols) located on the operation keys of consumer products such as cellular phones contribute to improving accessibility for all people, including people with visual impairment. The Japanese Standards Association (2000) standardized tactile dots and bars to ensure consistencies [1]. Additionally, the International Organization for Standardization (2011) also set a standard for tactile dots and bars, on the basis of the Japanese standard [2]. However, sufficient reliable data on the appropriate sizes and cross-section shapes of tactile dots and bars was not necessarily available. Therefore, more quantitative data for optimal dimensions of tactile dots and bars is required to revise existing standards and devise new standards. In this paper, we focused attention on cellular phones, which are becoming increasingly common globally, and evaluated influences of tactile-dot height and tip radius of curvature on the operational performance of cellular phones.

\section{Method}

The influences of height and tip radius of curvature of tactile dots on the operational performance of a cellular phone were measured by using an experi-

\footnotetext{
* Corresponding author. E-mail: wataru_toyoda@toki.waseda.jp 
mental apparatus including a mock-up of a cellular phone (Figures $1 \& 2$ ). The mock-up was connected with a personal computer through a data logger. Manipulation data was recorded in chronological order.

Tactile dots of different heights $(0.1,0.3,0.55$, and $0.75 \mathrm{~mm})$ and tip radii of curvature $(0.1,0.3,0.5,0.7$, and $0.9 \mathrm{~mm}$ ) were applied to the center of key 5 . The angle of skirts is 45 degrees which the skin tissue is not able to conform perfectly.

In the experiment, 16 younger (mean age 20.0 years, $\mathrm{SD}=1.2$ years) and 18 older (mean age 64.9 years, $\mathrm{SD}=2.9$ years) sighted persons as participants, with a hand covered by a curtain, manipulated the keys of the cellular phone with the thumb of their dominant hand while looking at 50 randomly ordered Arabic numbers per trial. Participants operated the mock-up four times in each of the 20 tactile-size conditions and without a tactile dot, for a total of 84 trials. Practice trials were conducted before starting the experiment. After each task, participants rated thumb pain during operation using a 5-point scale (1: Not

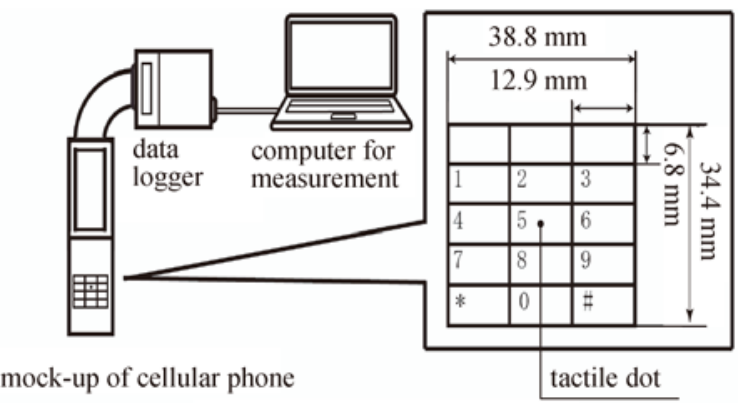

Fig. 1 Experimental apparatus

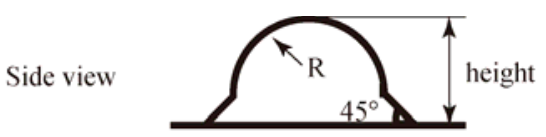

Fig. 2 A dimension of tactile dots used in the experiment painful - 5: Very painful). A complementary index was adopted to evaluate the subjective usability of tactile dots. All participants were without skin injuries or dermatosis and used a cellular phone with a 12-key numeric keypad on a regular basis. The influences of tactile-dot height and tip radius of curvature in both groups on operation time, error rate, and subjective thumb pain during operation was analyzed statistically using a two-factor repeated-measures analysis of variance (ANOVA). This study has obtained the permission from Ethical Committee on Human Research of Waseda University.

\section{Result}

ANOVA on all evaluation indices found significant interactions, in both group (Table 1). Overall, the younger group performed better in all size conditions than the older group. Furthermore, both groups tended to operate cellular phones more rapidly and accurately at a particular height with larger tip radius of curvature (Figures $2 \& 3$ ). As for height, younger participants performed better at $0.3 \mathrm{~mm}$ and relatively poorly at $0.1 \mathrm{~mm}$ and $0.75 \mathrm{~mm}$, whereas older participants tended to operate the phone better with $0.55 \mathrm{~mm}$ or more.

On the other hand, many older participants released their thumb from the keypad between key inputs in all conditions. Younger participants also operated this way with high tactile dots of $0.75 \mathrm{~mm}$ and often could not grip the cellular phone very well. However, at around $0.3 \mathrm{~mm}$, the younger operated the phone with their thumb touching the keypad continually.

In terms of pain, both groups felt more pain when tactile dots were higher and pointed. In particular, the average pain scores of the $0.75 \mathrm{~mm}$ height in the younger group were over about 3.6 and strongly painful.

Table 1 Results of a two-factor repeated-measures analysis of variance

\begin{tabular}{lcr}
\hline & Younger participants & $F(d f)$ \\
\hline & $3.28(12,180) *$ & $2.35(12,204) *$ \\
Operation time & $4.29(12,180) * *$ & $2.94(12,204) * *$ \\
Error rate & $7.99(12,180) * * *$ & $3.52(12,204) * *$ \\
Pain in the thumb during operations & $*: p<0.05, * *: p<0.01, * * *: p<0.001$
\end{tabular}




\section{Discussion}

Results show that a larger tip radius of curvature of tactile dots improved operational performance of cellular phones, because the dots distorted the user's fingertip more and are more perceivable.

The finding that appropriate height condition was different between young and older participants is attributed to differences in the behavior of the fingers. In addition, the tactile acuity decreases with aging (Manning \& Tremblay, 2006; Thornbury \& Mistretta, 1981) [3]. Therefore, the older people are easy to perceive the higher tactile dot and operate the cellular phone with that when operating.

In conclusion, larger tip radius of curvature dots is more suitable for practical use of cellular phones. Relatively high dots are useful for improving accessibility of products for the older. In contrast, as for younger participants, both too high and low height dots are not effective to improve the operational performance of cellular phones and there is an appropriate range of the height of tactile dots.

\section{References}

[1] International Organization for Standardization, ISO/DIS 24503: Ergonomics - Accessible design - Tactile dots and bars on consumer products, International Organization for Standardization, Geneva, 2011.

[2] Japanese Standards Association, JIS S 0011: Guidelines for all people including older and people with disabilities - making tactile dots on consumer products, Japanese Standards Association, Tokyo, 2000.

[3] H. Manning, F. Tremblay, Age differences in tactile pattern recognition at the fingertip, Somatosensory and Motor Research, Vol. 23, No. 3, 2006, pp. 147-155.

[4] J.M. Thornbury, C.M. Mistretta, Tactile Sensitivity as a Function of Age, Journal of Gerontology, Vol. 36, No. 1, 1981, pp. 34-39.
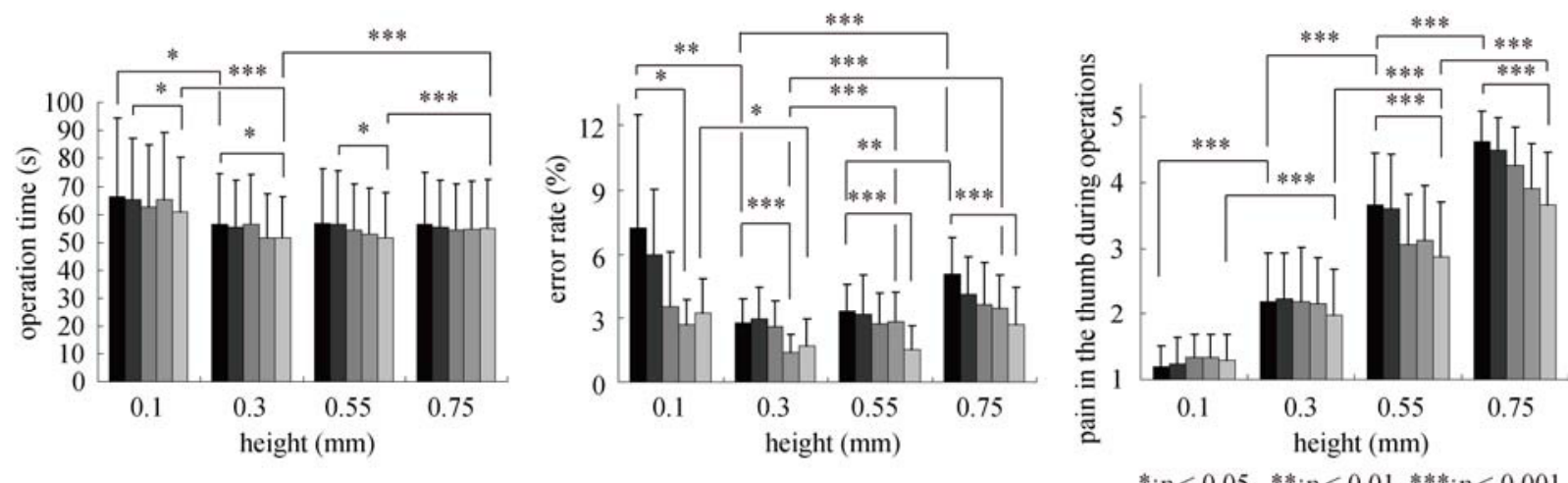

Tip radius of curvature $(\mathrm{mm})$

0.1

0.3

0.5

0.7

0.9

Fig. 3 Results of younger participants
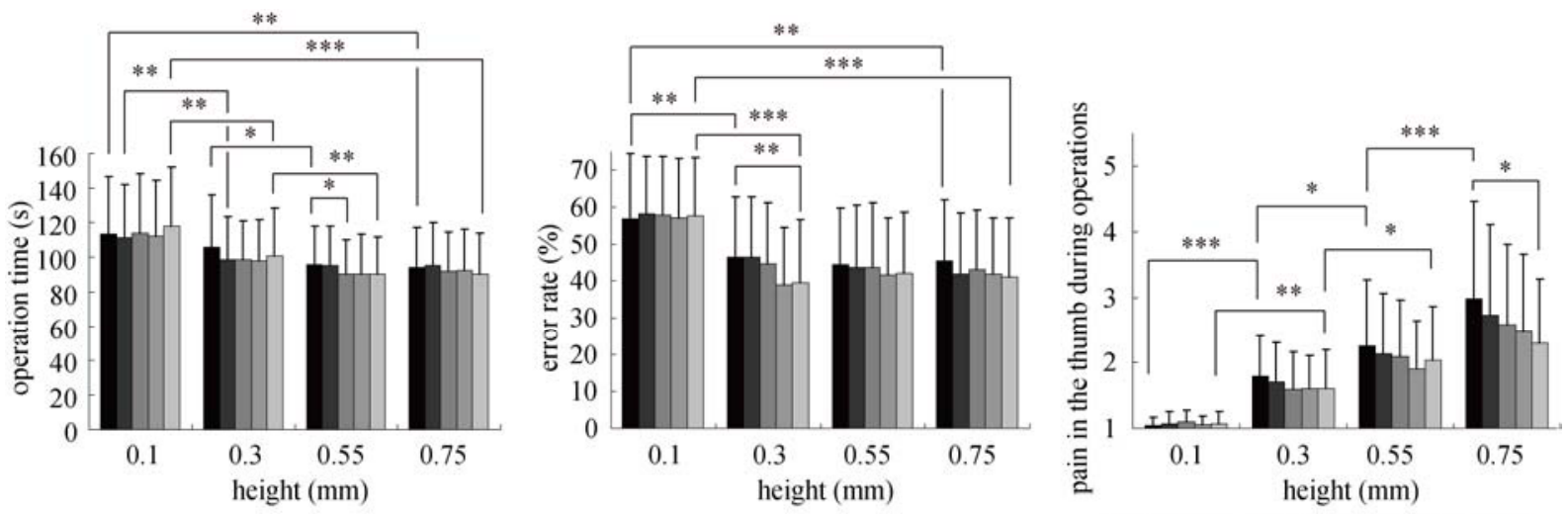

Tip radius of curvature $(\mathrm{mm})$

口 0.1

0.3

$*: p<0.05, * *: p<0.01, * * *: p<0.001$

Fig. 4 Results of older participants 